Pacific Journal of Mathematics

CENTRALIZERS OF TRANSITIVE SEMIGROUP ACTIONS AND 


\title{
CENTRALIZERS OF TRANSITIVE SEMIGROUP ACTIONS AND ENDOMORPHISMS OF TREES
}

\author{
Charles Wells
}

\begin{abstract}
A tree is locally finite if the interval between any two points is finite. A local isomorphism of a tree with itself is a homomorphism which is an isomorphism when restricted to any interval. Two theorems are proved. One characterizes those locally finite trees which have transitive automorphism groups, and those which have transitive local-isomorphism monoids. The other theorem gives necessary and sufficient conditions for a non-injective transformation to be centralized by a transitive permutation group, and necessary and sufficient conditions for it to be centralized by a transitive transformation semigroup. Also, an example is given of a nonlocally-finite tree with transitive automorphism group.
\end{abstract}

1. Preliminaries and statements of the theorems. All functions will be written to the right of the argument, and functional composition will read from left to right. If $\alpha: X \rightarrow X$ is a function, and $x \in X$, then $x \alpha^{-1}$ denotes the inverse image of $x$ under $\alpha$. If $X$ is any set, $|X|$ denotes the cardinality of $X$.

A semigroup $S$ acts on a set $X$ on the right if, for every $x \in X$ and $s \in S, x s$ denotes an element of $X$, and

$$
(x s) t=x(s t) \quad(x \in X, s \in S) .
$$

Then $X$ is an $S_{\text {- }}$-set or an $S$-operand.

An action by a semigroup $S$ on $X$ is transitive if for every $x, y \in X$, there is an element $s \in S$ such that $x s=y$. A subset $G$ of $X$ generates the $S$-set $X$ if for every $x \in X$ there is $g \in G$ and $s \in S$ such that $g s=x$. Thus an $S$-set is transitive if and only if every one-element subset of $X$ generates $X$.

Let $X$ and $Y$ be $S$-sets. A function $\alpha: X \rightarrow Y$ is an $S$ homomorphism (equivariant map) if

$$
(x \alpha) s=(x s) \alpha \quad(x \in X, s \in S) .
$$

$S$-endomorphisms and $S$-automorphisms are defined in the obvious way. It is easy to see that the $S$-endomorphisms of an $S$-set $X$ form a semigroup $\operatorname{End}_{S} X$ and the $S$-automorphisms form a group Aut $_{S} X$.

Let $T$ be a partially ordered set; its order relation, like all those in 
this paper, will be denoted $\leqq \quad T$ is a tree if the set $t A \underset{\text { def }}{=}\{u \in T: t<u\}$ is well-ordered (whenever it is nonempty) for every $t \in T . \quad T$ is nontrivial if $t A$ is nonempty for some $t \in T$. An element of $t A$ is an ancestor of $t$.

If $T$ is a tree, define for each $t \in T$ :

$t P=$ the parent of $t=$ the minimum of the set $t A-\{t\}$, if it exists.

$t B=t P P^{-1}=$ the set of siblings of $t$.

$t D=\{u \in T: u<t\}=$ the set of descendants of $t$.

$t C=t P^{-1}=$ the set of children of $t$.

Observe that $t C$ may be empty even if $t D$ is nonempty.

If $t, u \in T$, the interval from $t$ to $u$ is the set $[t, u]=\{x \in T: t \leqq x \leqq$ $u\}$; the notation will be used only when $t \leqq u$. T is locally finite if $[t, u]$ is a finite set for all $t, u \in T$ with $t \leqq u$. (This could be called vertical local finiteness, contrasted with horizontal local finiteness which would require that every sibling class be finite).

A filter of $T$ is a subset $X$ such that if $x \in X$ and $x<y$, then $y \in X . \quad X$ is an ideal of $T$ if $x \in X$ and $y<x$ implies $y \in X$.

Let $T$ and $T^{\prime}$ be trees. A bijection $f: T \rightarrow T^{\prime}$ is an isomorphism if $t \leqq u \Leftrightarrow t f \leqq u f$ for all $t, u \in T$. A function $g: T \rightarrow T^{\prime}$. is a local isomorphism if $g$ restricted to $[t, u]$ is an isomorphism from $[t, u]$ to $[t g, u g]$ for all $t, u \in T$ with $t \leqq u$. The set of isomorphisms of a tree $T$ with itself forms a group Aut $T$ under functional composition, and similarly the set of local isomorphisms of $T$ with itself forms a semigroup $L I(T)$.

Finally, let $\omega$ denote the set of nonnegative integers regarded as a well-ordered set.

In this paper, the following two theorems will be proved.

THEOREM 1. Let $T$ be a nontrivial locally finite tree. Then

(a) $L I(T)$ is transitive if and only if $T$ has no maximal or minimal elements.

(b) Aut $T$ is transitive if and only if $T$ has no maximal or minimal elements and $|t B|=|u B|$ for all $t, u \in T$.

THEOREM 2. Let $S$ be a semigroup and $X$ a transitive $S$-set with $S$-endomorphism $\alpha$. Then $\alpha$ is surjective, and if $\alpha$ is not injective, then $\alpha^{k}$ has no fixed points for any positive integer $k$. Conversely, if $\alpha: X \rightarrow X$ is a surjective function with the property that $\alpha^{k}$ has no fixed points for any positive integer $k$, then there is a transitive semigroup of functions from $X$ to $X$ with respect to which $\alpha$ is an endomorphism (in other words, $\alpha$ commutes with every function of the semigroup). This semigroup of functions can be taken to be a group of permutations if and only if $\left|x \alpha^{-1}\right|=\left|y \alpha^{-1}\right|$ for all $x, y \in X$. 
Observe that as a corollary of Theorem 2, a transitive semigroup action on a finite set can only be centralized by permutations.

2. Proof of Theorem 1. We need three lemmas.

LEMmA 1. Let $T$ be a tree. Then $T$ is locally finite and has no maximal elements if and only if $t A$ is order-isomorphic to $\omega$ for every $t \in T$.

Proof. Let $T$ be locally finite and have no maximal elements. Let $t \in T$. Define $\beta: \omega \rightarrow t A$ by

$$
\begin{aligned}
0 \beta & =t \\
(n+1) \beta & =(n \beta) P \quad(n=1,2, \cdots) .
\end{aligned}
$$

Then for $k \in \omega, k \beta$ is clearly $t P^{k}$. It is easy to see that $\beta$ is an injective, order-preserving map. It is also surjective: If $u \in t A$, then $[t, u]$ is finite, so that $u=t P^{k}$ for some integer $k$; hence $u=k \beta$.

The converse is obvious.

Lemma 2. Let $T$ and $T^{\prime}$ be trees, and $f: T \rightarrow T^{\prime}$ a function. Then the following are equivalent:

(a) $f$ is a local isomorphism.

(b) For all $t \in T, f$ restricted to $t A$ is an injective order-preserving function onto an initial segment of tfA.

(c) For all $t, u \in T$ and $w \in T^{\prime}$,

(i) $t<u \Rightarrow t f<u f$

and (ii) $t f<w<u f \Rightarrow$ there is $v \in T$ such that $t<v<u$ and $v f=w$.

Proof. The proof, particularly in the order (a) $\Rightarrow(c) \Rightarrow(b) \Rightarrow(a)$, is an easy consequence of the definitions of the terms involved and is omitted.

Lemma 3. Let $T$ be a tree, $t \in T$ and $f \in L I(T)$. Then $t f P=t P f$.

Proof. Immediate from Lemma 2(c).

We now prove the forward half of Theorem 1(a). Let $L I(T)$ be transitive. Since $T$ is nontrivial, there are $t, u \in T$ with $t<u$. If $x \in T$, there are $f, g \in L I(T)$ with $t f=x$ and $u g=x$. Then $\operatorname{tg}<x<u f$, so that $x$ is neither maximal nor minimal.

The forward half of Theorem 1(b) now follows, since if Aut $T$ is transitive, $L I(T)$ certainly is, and an automorphism restricted to a sibling set must be a bijection onto another sibling set. 
Now for the converse half of Theorem 1(b). Let $T$ be a locally finite tree with no maximal or minimal elements. Let $t, u \in T$. We must construct an automorphism $f$ of $T$ with $t f=u$. To do this, we first construct a sequence $f_{0}, f_{1}, f_{2}, \cdots$ of functions with increasing domains $D_{0}, D_{1}, \cdots$.

Take $f_{0}$ to be an order isomorphism from $t A$ to $u A$; it exists (in fact it is unique) by Lemma 1 , and clearly $t f_{0}=u$. The domain $D_{0}$ of $f_{0}$ is thus $t A$.

Let $D_{1}=D_{0} \cup\left(\cup_{x \in D_{0}} x B\right)$. For each $x \in D_{0}$, let $g_{x}: x B \rightarrow x f_{0} B$ be a bijection such that $x g_{x}=x f_{0}$. Define $f_{1}=f_{0} \cup\left(\cup_{x \in D_{0}} g_{x}\right)$. Then $f_{1}$ is well-defined because for $x, y \in D_{0}, x B$ and $y B$ are disjoint if $x \neq y$, and $x B$ intersects $D_{0}$ precisely in $\{x\}$.

It is not difficult to show that (3), (4) and (5) below hold for $i=1$.

(3) $D_{i-1} \subset D_{\imath}$ and $f_{\imath} \mid D_{t-1}=f_{i-1}$.

(4) $D_{\imath}$ and $D_{\imath} f_{\imath}$ are both filters of $T$.

(5) $f_{t}$ is an isomorphism from $D_{i}$ to $D_{t} f_{t}$.

Now suppose that $f_{t}$ has been defined with domain $D_{1}$ so that (3), (4) and (5) are true. Suppose also $i>0$.

We define $f_{t+1}$ and $D_{t+1}$ as follows. Let $M_{t}$ denote the set of minimal élements of $D_{i}\left(M_{i}\right.$ is nonempty, but we do not need that fact). Let $E_{\mathrm{t}}=\cup_{x \in M_{i}} x C ; E_{\mathrm{t}}$ is the set of immediate descendants of elements of $M_{\imath}$. It is important to note that $E_{\iota}$ is the disjoint union of the $x C$ 's.

For each $x \in M_{i}$, let $g_{x}: x C \rightarrow x f_{i} C$ be a bijection. (Note: $x C$ is nonempty because $[y, x]$ is finite for any $y<x$.) Let $D_{i+1}=D_{i} \cup E_{i}$, and $f_{i+1}=f_{t} \cup\left(\cup_{x \in M_{i}} g_{i}\right)$. Then $f_{t+1}$ is well-defined because of the fact that $E_{t}$ is a disjoint union of the $x C$ 's. It is straightforward to check (it involves numerous cases) that $f_{i+1}$ and $D_{i+1}$ satisfy (3) through (5) with $i+1$ instead of $i$.

Now let $f_{\omega}$ be the union of the functions $f_{i}$. Condition (3) implies that $f_{\omega}$ is well-defined, and conditions (4) and (5) that $f_{\omega}$ is an isomorphism between filters of $T$. Actually, more is true: The domain and the image of $f_{\omega}$ are each both filters and ideals of $T$. To see the latter, observe that if $x \in D_{t}$, then any element which is $k$ levels below $x$ will be included in $D_{i+k}$; thus every element below $x$ is included because of local finiteness. An analogous argument works for the image of $f_{\omega}$.

Furthermore, either $\operatorname{dom} f_{\omega}=\operatorname{im} f_{\omega}$ or they are disjoint. (In fact, they are connected components of $T$ ). For let $w, x, y \in \operatorname{dom} f_{\omega}$ and $x f_{\omega}=y$. By construction of $\operatorname{dom} f_{\omega}$, there is $z \in t A$ with $w \leqq z$ and $y \leqq z$. Since $\operatorname{im} f_{\omega}$ is both a filter and an ideal, it follows that $w \in \operatorname{im} f_{\omega}$. A similar argument shows that if $x$ and $y$ are as before and $w \in \operatorname{im} f_{\omega}$, then $w \in \operatorname{dom} f_{\omega}$.

It follows that either $f_{\omega}$ or $f_{\omega} \cup f_{\omega}^{-1}$ is an automorphism of a subtree $X$ 
such that if $t \in T-X$, then $t$ is unrelated to any element of $X$. Thus that automorphism can be safely extended to all of $T$ by stipulating that it be the identity on $T-X$. By construction it takes $t$ to $u$ as required.

Finally, to prove the converse half of Theorem 1(a), the preceding construction can be modified easily as follows: At the first stage, take $g_{x}: x B \rightarrow x f_{0} B$ to be any function with $x g_{x}=x f_{0}$. At each later stage, $g_{x}$ can be any function from $x C$ to $x f_{i} C$. Conditions (3) and (4) will still be true at each stage, and condition (5) will be true if the word "isomorphism" is replaced by the phrase "local isomorphism". The function $f$ thus constructed will be a local isomorphism from its domain to its image. This function (rather than $f_{\omega} \cup f_{\omega}^{-1}$ as in the automorphism case) may then be extended to all of $T$ by taking it to be the identity function outside the domain of $f_{\omega}$. The resulting function will then be a local isomorphism taking $t$ to $u$.

3. Proof of Theorem 2. The following two facts are needed for the proof of the forward part of Theorem 2. Both follow easily from (2). In both, $S$ is a semigroup.

Lemma 4. Let $X$ be an $S$-set with endomorphism $\alpha$. Then $\alpha$ is surjective if and only if $\operatorname{Im} \alpha$ generates $X$.

Lemma 5. Let $X$ be a transitive $S$-set with endomorphism $\alpha$. Then $\alpha$ has a fixed point if and only if $\alpha=i d_{X}$.

Now assume that $X$ is a transitive $S$-set with endomorphism $\alpha$. Then $\alpha$ is surjective by Lemma 4 , because every element of $X$ generates $X$. Furthermore, Lemma 5 applies equally well to $\alpha^{k}$ for any $k$. But no power of a noninjective function can be the identity function. This proves the forward part of Theorem 2 .

The converse part of Theorem 2 follows from Theorem 1. Suppose that $\alpha: X \rightarrow X$ is a surjective function with the property that no power of $\alpha$ has a fixed point. Define a relation $\leqq$ on $X$ this way:

(6) $x \leqq y$ if $y=x \alpha^{k}$ for some nonnegative integer $k$.

It is easy to see that $\leqq$ is reflexive and transitive. It is also antisymmetric: If $y=x \alpha^{k}$ and $x=y \alpha^{m}$ for some nonnegative integers $k$ and $m$, then $y$ is a fixed point of $\alpha^{k+m}$, which means that $k+m=0$, hence $k=m=0$, hence $x=y$. Thus $(X, \leqq)$ is a poset. Furthermore, if $x \in X$, then $x A$ is obviously isomorphic to the set $\omega$, so that $(X, \leqq)$ is a locally finite tree with no maximal elements by Lemma 1 .

It is immediate that $\alpha$ is the parent mapping of the tree $(X, \leqq)$. Since $\alpha$ is surjective, $(X, \leqq)$ has no minimal elements, so by Theorem 1 , $L I(T)$ is transitive. But by Lemma 3, every function in $L I(T)$ commutes with $\alpha$, as required. 
The last sentence of Theorem 2 clearly follows from Theorem 1(b).

4. Remarks. A congruence on an $S$-set $X$ is a partition $\pi$ of $X$ with the property that if $u \in \pi$ and $u, v \in U$, then $u s$ and $v s$ are in the same block of $\pi$ for any $s \in S$. If $X$ has an $S$-endomorphism $\alpha$, the set $\left\{x \alpha^{-1} \mid x \in X\right\}$ is a congruence on $X$ (this follows easily from (2)). It follows that if $X$ is a transitive $S$-set with no nontrivial congruences, then $\alpha$ must be a permutation of $X$. In particular, a primitive group action, finite or infinite, is centralized only by permutations.

It is easy to see, using (2), that a doubly transitive semigroup action is centralized only by the identity function.

The way to construct permutations that centralize finite transitive group actions has been known since the turn of the century. A recent exposition is in Kerber [2]. Expositions of the elementary theory of semigroup actions may be found in Banaschewski [1], Knauer [3], Petrich [5] and Wells [8] with varying emphases. This subject is treated from an axiomatic point of view by Saìn [6].

Infinite trees have been used as tools in various areas, for example in mathematical linguistics. References may be found in Maxson [4]. Serre [7] describes the structure of groups acting on trees. (Warning: My terminology is quite different from his!).

It would be interesting to characterize all the trees with transitive automorphism semigroup. Such a tree need not be locally finite; the only examples I know, however, are all quite complicated. The one which can be described most succinctly is the following:

Let $T$ consist of all ordered triples $(a, b, \gamma)$ with $a, b \in Z$ ( $Z$ the set of all integers) and $\gamma: Z \rightarrow Z$ a function for which

$$
n \gamma=0 \text { for } n<b
$$

Define

$$
(a, b, \gamma) \leqq\left(a^{\prime}, b^{\prime}, \gamma^{\prime}\right) \Leftrightarrow\left\{\begin{array}{c}
a \leqq a^{\prime}, b=b^{\prime} \text { and } \gamma=\gamma^{\prime} \\
\text { or } b<b^{\prime},\left(b^{\prime}-1\right) \gamma \leqq a^{\prime} \text { and } \\
n \gamma=n \gamma^{\prime} \text { for all } n \geqq b^{\prime}
\end{array}\right.
$$

A tedious but straightforward proof shows that $T$ is a nonlocally finite tree. The element $(a, b, \gamma)$ may be visualized as the integer $a$ in a copy of $Z$ which is hanging down from the element $\left(b \gamma, b+1, \gamma^{\prime}\right)$, where $\gamma^{\prime}$ agrees with $\gamma$ except that $b \gamma^{\prime}=0$. Each element of $T$ has a copy of $Z$ hanging below it.

If $(a, b, \gamma)$ and $\left(a^{\prime}, b^{\prime}, \gamma^{\prime}\right)$ are elements of $T$, the following defines an automorphism $\varphi$ of $T$ which takes $(a, b, \gamma)$ to $\left(a^{\prime}, b^{\prime}, \gamma^{\prime}\right)$.

Let $\eta: T \rightarrow T$ be defined by 


$$
y \eta=\left\{\begin{array}{l}
\left(y+b^{\prime}-b-1\right) \gamma^{\prime}-(y-1) \gamma \text { for } y>b . \\
a^{\prime}-a \text { for } y \leqq b .
\end{array}\right.
$$

If $(x, y, \xi) \in T$, define $\bar{\xi}: Z \rightarrow Z$ by

(10) $n \bar{\xi}=\left\{\begin{array}{l}\left(n+b-b^{\prime}\right) \xi+n \gamma^{\prime}-\left(n+b-b^{\prime}\right) \gamma \text { for } n \geqq y+b^{\prime}-b \\ \text { and } n \geqq b^{\prime} . \\ \left(n+b-b^{\prime}\right) \xi+a^{\prime}-a \text { for } n \geqq y+b^{\prime}-b \text { and } n<b^{\prime} . \\ 0 \quad \text { for } n<y+b^{\prime}-b .\end{array}\right.$

Now set, for $(x, y, \xi) \in T$,

$$
(x, y, \xi) \varphi=\left(x+y \eta, y+b^{\prime}-b, \bar{\xi}\right) .
$$

Then $\varphi$ will be an automorphism of $T$ taking $(a, b, \gamma)$ to $\left(a^{\prime}, b^{\prime}, \gamma^{\prime}\right)$.

The author wishes to thank Steve McCleary for pointing out an error in the original version of Theorem 1, and Paul Kainen, who came up with the idea behind the preceding example.

\section{REFERENCES}

1. B. Banaschewski, Functions into categorles of $M$-sets, Abh. Math. Sem. Univ. Hamburg, 38 (1072), 49-64.

2. A. Kerber. Representations of permutation groups I, Lecture Notes in Mathematıcs 240. Springer Verlag, 1971.

3. U. Knauer, Projectivity of acts and Morita equivalence of monoids, Semigroup Forum, 3 (1971/72), 359-370.

4. C. Maxson, Semigroups of order-preserving partial endomorphisms on trees, I, To be published.

5. M. Petrich, Dense extensions of semigroups I, J. reıne ang. Math., 258 (1973), 103-125, section 2.

6. B. M. Šain, Transformattve semigroups of transformations, Mat. Sbornik (N.S.) 71 (113), (1968), $65-82$.

7. J. P. Serre, Arbes, Amalgams et $S L_{2}$. Mimeographed notes.

8 C. Wells, Some applications of the wreath product construction, Amer. Math. Monthly 83, May, 1976.

Received March 18, 1975.

Eidgen Technische Hochschule

AND

Case Western Reserve University 




\section{Pacific Journal of Mathematics}

Vol. 64, No. 1

May, 1976

Walter Allegretto, Nonoscillation theory of elliptic equations of order $2 n \ldots \ldots \quad 1$

Bruce Allem Anderson, Sequencings and starters.................. 17

Friedrich-Wilhelm Bauer, A shape theory with singular homology .......... 25

John Kelly Beem, Characterizing Finsler spaces which are

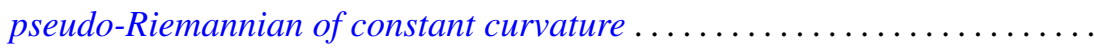

Dennis K. Burke and Ernest A. Michael, On certain point-countable

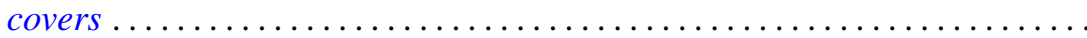

Robert Chen, A generalization of a theorem of Chacon ............... 93

Francis H. Clarke, On the inverse function theorem ................ 97

James Bryan Collier, The dual of a space with the Radon-Nikodým

property ....................................... 103

John E. Cruthirds, Infinite Galois theory for commutative rings ............ 107

Artatrana Dash, Joint essential spectra......................... 119

Robert M. DeVos, Subsequences and rearrangements of sequences in FK

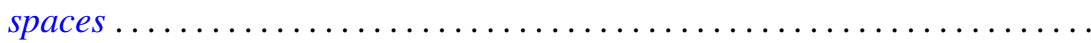

Geoffrey Fox and Pedro Morales, Non-Hausdorff multifunction generalization

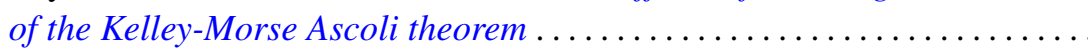

Richard Joseph Fleming, Jerome A. Goldstein and James E. Jamison, One

parameter groups of isometries on certain Banach spaces.............

Robert David Gulliver, II, Finiteness of the ramified set for branched

immersions of surfaces

Kenneth Hardy and István Juhász, Normality and the weak cb property ...... 167

C. A. Hayes, Derivation of the integrals of $L^{(q)}$-functions.

Frederic Timothy Howard, Roots of the Euler polynomials .

Robert Edward Jamison, II, Richard O'Brien and Peter Drummond Taylor, On

embedding a compact convex set into a locally convex topological vector space ....................................

Andrew Lelek, An example of a simple triod with surjective span smaller than span ...

Janet E. Mills, Certain congruences on orthodox semigroups

Donald J. Newman and A. R. Reddy, Rational approximation of $e^{-x}$ on the positive real axis.

John Robert Quine, Jr., Homotopies and intersection sequences ...

Nambury Sitarama Raju, Periodic Jacobi-Perron algorithms and fundamental units ....

Herbert Silverman, Convexity theorems for subclasses of univalent functions. . .

Charles Frederick Wells, Centralizers of transitive semigroup actions and endomorphisms of trees.........................

Volker Wrobel, Spectral approximation theorems in locally convex spaces ..................... 